\title{
Avaliação da Motivação da Aprendizagem de Programação Avançada através de PBL
}

\author{
Francisco T. S. S. Pereira ${ }^{1}$, Roberto A. Bittencourt ${ }^{1}$ \\ ${ }^{1}$ UEFS -- Universidade Estadual de Feira de Santana \\ Av. Transnordestina, $\mathrm{s} / \mathrm{n}$, Novo Horizonte \\ Feira de Santana -- BA, Brasil -- 44036-900 \\ franncisco.p@gmail.com, roberto@uefs.br
}

\begin{abstract}
This paper assesses student motivation in an integrated course of object-oriented programming, data structures and software design that uses the problem-based learning (PBL) as a teaching-learning approach. This work replicates a previous analysis and the results show that motivation remains high during students' problem solving. In addition, motivation in the accompanying lectures directly reflects the results students achieved.
\end{abstract}

Resumo. Este trabalho apresenta uma avaliação da motivação em uma disciplina integrada de programação orientada a objetos, estruturas de dados e projeto de sistemas que utiliza a metodologia de aprendizagem baseada em problemas (PBL) como abordagem de ensino-aprendizagem. Este trabalho replica uma análise anterior e os resultados apontam que a motivação se mantém elevada durante a resolução dos problemas pelos estudantes. Além disso, a motivação nos módulos teóricos que acompanham os problemas $P B L$ reflete diretamente os resultados obtidos pelos estudantes.

\section{Introdução}

Evasão e reprovação nos primeiros semestres de cursos de TI são um tema comum na área da educação em computação. Podem ser causados pelas dificuldades de aprendizagem típicas, mas também pela desmotivação dos estudantes para aprender programação, que muitas vezes pode parecer difícil e abstrata.

$\mathrm{O}$ estudo dos fatores não cognitivos que influenciam no aprendizado do estudante é bastante importante para a redução dos altos índices de reprovação. O estilo de aprendizagem e a motivação interferem diretamente no aprendizado [Jenkins 2002]. O primeiro diz a respeito a como o estudante estuda, quais metodologias são utilizadas e a importância do papel do professor nesta abordagem, que deve assegurar que o estudante esteja no caminho certo. O segundo fator, a motivação, é um dos fatores fundamentais para a explicação da direção e a magnitude do comportamento do ser humano em relação aos objetivos que as pessoas escolhem alcançar [Keller 2009].

No ensino da segunda disciplina de programação no ensino superior (também chamada de CS2 na literatura), em que estudantes já aprenderam previamente conceitos de programação, é comum encontrar relatos de que a maioria das adversidades encontradas envolvem a dificuldade em transformar um problema do papel para o código e não de entender os conceitos de programação propriamente ditos. Por isso, 
professores buscam encontrar melhores soluções para motivar o estudante no processo de aprendizagem [Arshad 2009]. Além disso, metodologias ativas, como a Aprendizagem Baseada em Problemas (PBL, do termo em inglês), são bastante utilizadas para introduzir os estudantes a problemas que se relacionam com a vida real, buscando estimular a autonomia, trabalho em grupo, expressão oral e colaboração do estudante [Angelo et al. 2014].

Com o objetivo de aumentar o acervo de estudos dos aspectos motivacionais e seu impacto nos resultados de aprendizagem, o presente artigo busca analisar a motivação dos estudantes nas as disciplinas do CS2 da Universidade Estadual de Feira de Santana (UEFS), que formam um curso integrado de Programação Orientada a Objetos, Estruturas de Dados e Projeto de Sistemas. O presente trabalho replicou, no segundo semestre de 2019, outro trabalho realizado previamente [Ribeiro et al. 2018].

\section{Fundamentação Teórica}

\subsection{Motivação}

A motivação é um dos principais fatores que interferem diretamente na aprendizagem do estudante. Diversos autores apresentam a motivação como as vontades, necessidades, desejos e compulsões para participar e obter sucesso no processo de aprendizagem [Bergin e Reilly 2005, Keller 2009]. Apresentam ainda os conceitos de motivação intrínseca e extrínseca. O primeiro explica que indivíduos se comprometem em tarefas pelas recompensas que vêm diretamente dela. Ou seja, para um aluno que escolheu cursar computação, o curso em si já é considerado uma boa recompensa e é satisfatório motivacionalmente. O segundo explica que os indivíduos se comprometem em tarefas por suas recompensas posteriores. Por exemplo, um estudante poderá se sentir realizado com uma carreira, o que só é possível de ocorrer a partir da conclusão do curso.

Visando entender os fatores que influenciam a motivação de um estudante, Keller (2009) criou o modelo ARCS. Este modelo possui quatro categorias que estão relacionadas diretamente com a motivação do ser humano quanto à aprendizagem. A primeira categoria, Atenção, está diretamente ligada à estimulação da aprendizagem do estudante e à capacidade de obter interesse no assunto. A segunda, Relevância, diz respeito à crença do estudante de que a experiência de aprendizagem é relevante para seus objetivos. A terceira, Confiança, diz respeito à percepção do estudante de acreditar que ele obterá sucesso a partir da aprendizagem. Por fim, a quarta, Satisfação, tende a reforçar o contentamento que os estudantes sentem com o aprendizado, levando-os a querer continuar aprendendo para alcançar os seus objetivos pessoais.

\subsection{Programação Orientada a Objetos}

A Programação Orientada a Objetos (POO) é um paradigma bastante utilizado para o ensino de CS2 nas universidades. É comum ser ensinada usando a linguagem de programação Java, pela vasta quantidade de materiais e ferramentas de aprendizagem disponíveis. O ensino de POO é comumente realizado no Brasil após o aprendizado do estudante do paradigma imperativo procedural. Porém, Kölling (1999) sugere que, por causa das dificuldades encontradas pelos estudantes com a transição de paradigmas, esta deve ser estudada desde o início. De qualquer modo, a transição entre paradigmas costuma provocar dificuldades entre os estudantes [Ribeiro et al. 2018]. 


\subsection{Aprendizagem Baseada em Problemas}

A aprendizagem baseada em problemas (PBL - Problem Based Learning) é uma metodologia centrada no estudante desenvolvida por Howard Barrows. Nela, o estudante tem a oportunidade de desempenhar papéis ativos no processo de aprendizagem, decidindo o que estudar e assumindo responsabilidades diante do seu grupo de estudo [Angelo et al. 2014]. A metodologia foi inicialmente adotada em cursos de Medicina. Porém, atualmente, diversas áreas do conhecimento a adotam para o ensino, como é o caso da computação [Cintra e Bittencourt 2015, Angelo et al. 2014]. A partir de reuniões chamadas de sessões tutoriais, estudantes procuram resolver um problema em grupo, contando com um tutor facilitador no processo.

Em nosso modelo de PBL [Cintra e Bittencourt 2015], em um primeiro contato do grupo com o problema, deve-se realizar uma sessão de brainstorming onde ideias para a solução do problema serão discutidas. Após a discussão, é necessário sistematizar as ideias centrais, levantar questões para aprendizagem e traçar metas até o próximo encontro do grupo. Assim, em cada sessão são discutidas novas ideias apresentadas pelos estudantes, fatos conhecidos após estudo, questões apontadas durante a sessão, e metas traçadas para resolver o problema, ciclo repetido até chegar à sua solução.

\section{Metodologia}

Este estudo foi realizado na Universidade Estadual de Feira de Santana (UEFS), com os estudantes de Engenharia de Computação. Este curso usa PBL como uma de suas principais metodologias de ensino-aprendizagem. Geralmente, atividades com PBL ocorrem nos componentes curriculares denominados Estudos Integrados (EI).

Os EI promovem a interligação de disciplinas do curso a partir da organização em módulos teóricos (MT) e módulo integrador (MI). Em cada módulo teórico, conceitos específicos são abordados. No módulo integrador, o estudante integra os conceitos de todos os módulos através da solução de problemas no estilo PBL.

\subsection{Organização do Estudo Integrado}

No segundo semestre do curso, e Estudo Integrado de Programação integra, através do Módulo Integrador (MI) de Programação, os conceitos discutidos nos módulos teóricos de Algoritmos e Programação II, Estruturas de Dados, Projetos de Sistemas. O primeiro MT apresenta os conceitos da linguagem de programação Java juntamente com o paradigma orientado a objetos. O segundo expõe os conceitos de listas, filas, árvores, grafos, dentre outras estruturas de dados. O terceiro discute conceitos de projeto de sistemas usando diagramas UML, além de testes de unidade e de integração. No Módulo Integrador (MI) de Programação, estudantes resolvem, através da metodologia PBL, três problemas fracamente estruturados e baseados em problemas do mundo real em um período entre três e cinco semanas para cada problema. Ao final, entregam um relatório técnico e o código-fonte e documentação do sistema desenvolvido.

\subsection{Participantes}

Participaram desta pesquisa os estudantes de Engenharia de Computação que cursaram o EI de Programação no segundo semestre de 2019. As turmas foram formadas por estudantes cursando as disciplinas pela primeira vez assim como alguns previamente 
reprovados, distribuídos da seguinte forma: Algoritmos e Programação - 43 estudantes (36 estudantes do sexo masculino e 7 do feminino), Estruturas de Dados - 45 estudantes (38, masculino, e 7, feminino), Projeto de Sistemas - 51 estudantes (43, masculino, e 8, feminino) e MI - Programação - 47 estudantes (40, masculino, e 7, feminino). Tivemos 52 participantes, levando em conta que os estudantes podem estar matriculados em um ou mais módulos, sendo que 43 estavam matriculados em todos os módulos.

\subsection{Coleta e Análise de Dados}

Antes de iniciar a coleta dos dados, para atender às questões de ética em pesquisa com seres humanos, os participantes assinaram um Termo de Consentimento Livre e Esclarecido (TCLE), garantindo o seu anonimato. Para mensurar motivação, usamos dois questionários fundamentados nos conceitos do modelo ARCS. No MI de Programação, usamos o questionário IMMS (Instructional Materials Motivation Survey), que procura medir reações para materiais de instrução autodirigidos. Nos módulos teóricos, usamos o CIS (Course Interest Survey), concebido para medir as reações dos estudantes diante de instrução guiada por um professor.

Para a coleta dos dados, os estudantes responderam a sete questionários. $\mathrm{O}$ primeiro foi um questionário demográfico no início do semestre. Ao final de cada uma das três unidades, responderam a um questionário IMMS no MI de Programação para avaliar a motivação dos estudantes durante a resolução do problema. Além disso, para cada módulo teórico, responderam a um questionário CIS no final do semestre letivo.

Também foram coletadas as notas dos estudantes durante o semestre letivo. Para a disciplina MI - Programação, a coleta foi realizada ao final de cada unidade. Já para os módulos teóricos, somente a nota final do aluno foi coletada. Os resultados foram analisados utilizando estatísticas descritivas, testes de hipótese e correlação. Estatísticas, paramétricas ou não, foram usadas de acordo com a normalidade ou não dos dados.

\section{Resultados}

A partir da coleta e análise dos dados, reunimos inicialmente os dados dos questionários IMMS e CIS. Para cada questionário, pode-se analisar separadamente cada categoria do modelo ARCS: Atenção, Relevância, Confiança e Satisfação.

\subsection{Motivação no Módulo Integrador}

A Figura 1 apresenta os box-plots da média das categorias ARCS, computados pela média das três unidades de cada categoria para cada respondente. Mostramos apenas as médias pois o comportamento de cada categoria foi similar em todos as unidades.

Nota-se que a mediana da relevância foi maior em comparação com as outras categorias. Em contrapartida, a confiança obteve os menores valores da mediana. Porém, todas os resultados foram positivos, com mediana acima do valor neutro (3).

O teste de Friedman foi utilizado para verificar se o escore das quatro categorias varia significativamente entre os problemas, mostrando que os escores das categorias ARCS foram os mesmos com os seguintes parâmetros de $\chi^{2}$ para as comparações múltiplas entre problemas: 2,141, 2,487, 2,541 e 2,541, todos com valores de $p>0,05$. 


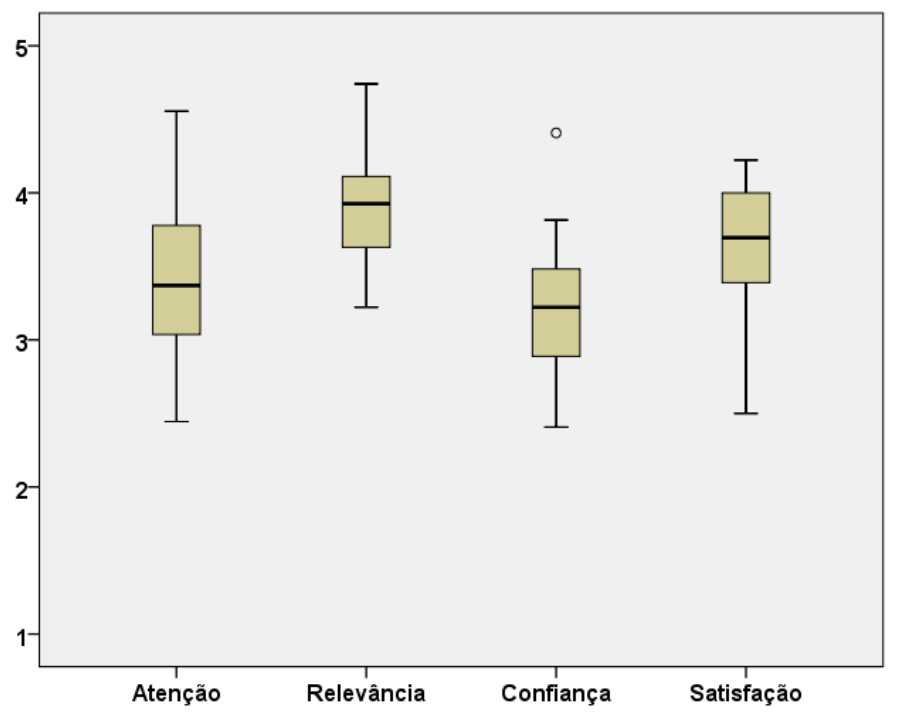

Figura 1. Box-plots das medias das categorias ARCS nos Problemas do MI

\subsection{Motivação nos Módulos Teóricos}

Em relação aos módulos teóricos, a Figura 2 apresenta os seguintes box-plots para cada categoria do ARCS. Estruturas de Dados apresenta uma maior dispersão nos dados nas categorias Atenção, Relevância e Confiança. Já Algoritmos e Programação II, apresenta maior dispersão em Satisfação. Para a categoria Atenção, somente o módulo Projeto de Sistemas obteve mediana acima de 3,0. Utilizamos ANOVA para testar se a Atenção varia significativamente entre os módulos teóricos. Esta hipótese foi confirmada com $\mathrm{F}$ $=7,677, \mathrm{p}=0,001$. O teste post-hoc de Tukey demonstrou que os resultados apresentam diferenças significativas entre Projeto de Sistemas e Algoritmos e Programação II.

Para Relevância, somente o módulo Estruturas de Dados obteve a mediana acima de 3,0. Utilizamos ANOVA para testar se a Relevância varia significativamente entre os módulos teóricos, o que foi confirmado com $\mathrm{F}=5,323, \mathrm{p}=0,009$. O Teste posthoc de Tukey demonstrou que os resultados apresentaram diferenças significativas entre os módulos teóricos de Estruturas de Dados e Projeto de Sistemas.

Para Confiança, novamente o módulo Estruturas de Dados apresenta uma mediana acima de 3,0, ao contrário das outros módulos. Utilizamos ANOVA para testar se a Confiança varia significativamente entre os módulos teóricos, o que foi confirmado com $\mathrm{F}=13,859, \mathrm{p}<0.001$. O Teste post-hoc de Tukey demonstrou que os resultados apresentaram diferenças significativas entre: a) Projeto de Sistemas e Estruturas de Dados e b) Projeto de Sistemas e Algoritmos e Programação II.

Por fim, para a categoria Satisfação, o módulo Estruturas de Dados obteve uma mediana acima do valor neutro. Utilizando ANOVA para testar se a Satisfação varia significativamente entre os módulos teóricos, confirmamos isto com $\mathrm{F}=10,596, \mathrm{p}=$ 0,000195. O Teste post-hoc de Tukey demonstra que os resultados apresentaram diferenças significativas entre os módulos teóricos: a) Projeto de Sistemas e Estruturas de Dados e b) Projeto de Sistemas e Algoritmos e Programação II. 

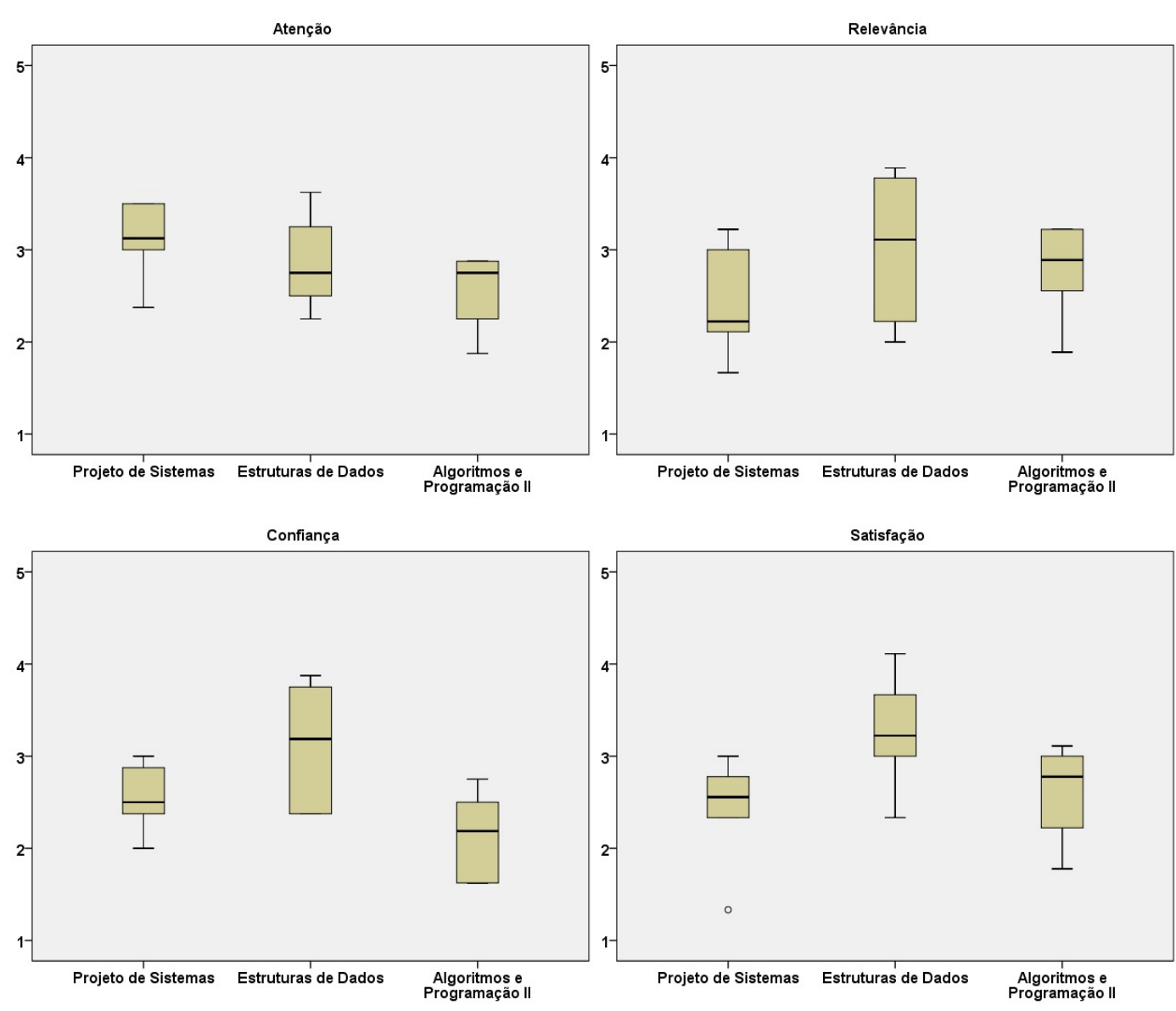

Figura 2. Box-plots das categorias ARCS nos módulos teóricos

\subsection{Relação entre Motivação e Aprendizagem}

A relação entre motivação e aprendizagem é apresentada a seguir, separados por módulo integrador e módulos teóricos.

\subsubsection{Módulo Integrador}

A Tabela $1^{1}$ apresenta as correlações entre as notas de cada problema dos estudantes no Módulo Integrador com as notas das categorias do ARCS de cada problema.

\begin{tabular}{ccccc}
\hline Problema & Atenção & Relevância & Confiança & Satisfação \\
\hline P1 & $-0,009(0,958)$ & $0,015(0,930)$ & $0,405^{*}(0,013)$ & $0,139(0,412)$ \\
\hline P2 & $0,126(0,451)$ & $0,453^{* *}(0,004)$ & $-0,096(0,568)$ & $0,218(0,189)$ \\
\hline P3 & $-0,078(0,718)$ & $0,227(0,286)$ & $0,171(0,423)$ & $0,463^{*}(0,023)$ \\
\hline
\end{tabular}

Tabela 1. Correlação entre Motivação e Aprendizagem no Módulo Integrador

\footnotetext{
${ }^{1} \mathrm{O}$ símbolo * indica que a correlação é significativa no nível 0,05 nas duas extremidades, enquanto que
} ** indica que a correlação é significativa no nível 0,01 nas duas extremidades. 
No Problema 1, percebe-se que não existe correlação entre a nota do estudante com Atenção e Relevância, mas existe uma correlação fraca com Satisfação e moderada com Confiança. Já no Problema 2, não existe correlação entre as notas e Confiança, mas existe uma correlação fraca com Atenção e Satisfação, e moderada com Relevância. No Problema 3, não existe correlação com Atenção; por outro lado, existe uma correlação fraca com Confiança e Relevância e moderada com Satisfação.

\subsubsection{Módulos Teóricos}

A Tabela 2 apresenta as correlações entre as notas finais dos estudantes nos Módulos Teóricos com os escores das categorias do ARCS nos respectivos módulos teóricos.

\begin{tabular}{ccccc}
\hline $\begin{array}{c}\text { Módulo } \\
\text { Teórico }\end{array}$ & Atenção & Relevância & Confiança & Satisfação \\
\hline $\begin{array}{c}\text { Algor. e } \\
\text { Programação II }\end{array}$ & $0,287(0,299)$ & $0,008(0,979)$ & $-0,076(0,788)$ & $0,014(0,961)$ \\
\hline $\begin{array}{c}\text { Estruturas de } \\
\text { Dados }\end{array}$ & $-0,016(0,949)$ & $-0,017(0,945)$ & $-0,077(0,760)$ & $0,324(0,190)$ \\
\hline Projeto de Sistemas & $0,673 *(0,023)$ & $0,243(0,471)$ & $0,500(0,117)$ & $0,371(0,261)$ \\
\hline
\end{tabular}

Tabela 2. Correlação entre Motivação e Aprendizagem nos Módulos Teóricos

Algoritmos e Programação II não apresenta correlação com Confiança, Relevância e Satisfação; por outro lado, apresenta uma correlação fraca com Atenção. Já Estruturas de Dados, não apresenta correlação com Atenção, Relevância e Confiança, porém apresenta correlação fraca com Satisfação. Por fim, Projeto de Sistemas apresenta uma correlação fraca com Relevância e Satisfação e forte com Confiança e Atenção.

\section{Discussão}

Um fato a ser observado entre as quatro categorias de motivação do ARCS (Atenção, Relevância, Confiança e Satisfação) é que todas as medianas alcançaram valores maiores que o valor neutro 3,0. Isso demonstra que todos os problemas foram bem recebidos pelos estudantes.

Em termos de Relevância, percebemos que ela sempre possui os maiores valores entre as categorias. A partir do conceito de relevância [Keller 2009] como um fator poderoso que pode determinar se a pessoa está motivada a aprender, pode-se fazer a associação entre esta definição com as respostas obtidas anteriormente. $O$ comportamento da mediana da relevância tende a se repetir em todas as unidades, pelo fato de o assunto abordado no módulo ser o assunto central da área onde está situado o seu curso. Logo, o estudante tende a achá-lo relevante.

Por outro lado, a Confiança apresenta os valores de mediana mais baixos quando comparado com as outras categorias. Isso é possivelmente explicado pelo fato do estudante nem sempre se sentir confiante com determinados assuntos, seja por causa da metodologia do professor ou por problemas pessoais que possam interferir na compreensão do conteúdo. 
A mediana da Atenção obteve resultados positivos (acima de três) em todos os problemas. Isso sugere que a organização e a aplicação dos problemas foram satisfatórias para os estudantes, capturando o interesse dos estudantes e estimulando a curiosidade para aprender. [Keller 2009].

Por fim, a Satisfação também obteve resultados da mediana acima da neutralidade. Para Keller (2009), a Satisfação deve ser um dos fatores principais para o estudante conquistar o desejo de continuar aprendendo. A Satisfação pode vir a partir de fatores intrínsecos e extrínsecos. A partir das conquistas obtidas durante o semestre, $\mathrm{o}$ estudante pode realçar a sua autoestima e, consequentemente, os seus sentimentos de competência e satisfação.

Ao observar a Figura 2, percebemos valores de Atenção maiores para os módulos Projeto de Sistemas e Estruturas de Dados e mais baixos para Algoritmos e Programação II. Isso pode se dar pelo fato de os assuntos de Projeto de Sistemas estarem mais relacionados com o projeto de um sistema desde o seu início, a partir da criação de diagramas, assunto que pode trazer a atenção do estudante pela proximidade com o mundo real, onde o desenvolvedor terá que projetar um software antes de desenvolvê-lo.

Os valores de Relevância foram maiores para Estruturas de Dados e Algoritmos e Programação II, somente o primeiro alcançando mediana acima do neutro. Uma justificativa possível para este comportamento é que os dois módulos utilizarem mais a programação do que Projeto de Sistemas. Esta última pode se tornar menos relevante para aqueles que acham que a programação é o aspecto mais importante do estudo integrado e não o design dos sistemas.

Em relação à Confiança, novamente Estruturas de Dados alcança o seu valor de mediana acima de três, seguida de Projeto de Sistemas e Algoritmos e Programação II. O baixo valor de mediana alcançado em Algoritmos e Programação II pode ter como explicação o novo paradigma de orientação a objetos que pode se tornar complexo para muitos que trabalharam anteriormente com o paradigma procedural.

Por fim, em termos de Satisfação, novamente o módulo de Estruturas de Dados alcançou mediana acima de três, seguido por Algoritmos e Programação II e, por fim, Projeto de Sistemas. Um fato a ser observado no módulo de Projeto de Sistemas é que os estudantes ficaram sem professor na disciplina por um certo período, o que pode ter prejudicado a experiência na disciplina. Alguns módulos obtiveram resultados melhores que os outros, o que pode ser explicado também pela diferença da metodologia de ensino dos professores, que pode impactar de forma positiva ou negativa na motivação do estudante.

\subsection{Relação entre Motivação e Aprendizagem}

Após computar os escores do Atenção, Relevância, Confiança e Satisfação, foi importante verificar a correlação entre as categorias do ARCS e as notas dos estudantes nos módulos teóricos e integrador.

Verificamos que o Problema 1 não obteve correlação entre a Atenção e Relevância, mostrando que, independentemente da Atenção e Relevância, os estudantes tinham boas notas. O oposto acontece com a Confiança, que obteve uma maior correlação. Esse resultado é provavelmente explicado pelo fato de o primeiro problema 
ser novidade para os estudantes, trazendo consigo novos assuntos e uma nova linguagem de programação, além da facilidade maior, por ser o primeiro problema, levando-os a altos níveis de confiança.

Já no Problema 2, nota-se que a Relevância obteve a maior correlação, provavelmente pelo fato de o assunto central neste problema ter sido Árvore Binária, assunto que desperta o interesse do estudante pelo fato de os algoritmos de balanceamento serem desafiadores.

Por fim, o Problema 3 traz consigo os conceitos de interface gráfica e grafos. Houve uma forte correlação com a Satisfação neste problema, possivelmente pelo fato de o mesmo ter sido o último do semestre e a utilização da interface gráfica substituindo a interface de linha de comando potencializar a satisfação dos estudantes.

Em Algoritmos e Programação II, tem-se uma maior correlação entre a nota do estudante e a Atenção, possivelmente explicado pelo fato de os estudantes serem considerados majors e a matéria apresentar assuntos importantes para o curso.

Já em Estruturas de Dados, houve somente correlação positiva com a Satisfação, sugerindo que o conteúdo ministrado na disciplina, juntamente com a metodologia introduzida, tornaram os estudantes satisfeitos com o aprendizado, motivando-os a continuar aprendendo.

Por fim, em Projeto de Sistemas, houve correlação em todas as categorias, especialmente atenção e confiança, o que, por motivos da forte ligação entre o aprendizado na universidade com os projetos realizados na vida real, podem ter chamado a atenção dos alunos e garantiram a sua dedicação nos assuntos deste módulo.

\subsection{Ameaças a validade}

Durante o final do período letivo 2019.2, que se encerrou em março de 2020, foi declarada a pandemia do coronavírus, o que pode considerada como ameaça à validade interna por prejudicar a obtenção de respostas aos questionários finais para as matérias do módulo teórico, pelo fato de o número de respostas aos questionários ter sido menor que o esperado, e também potencialmente afetando os resultados finais dos estudantes.

Em termos de validade externa, este artigo utilizou uma metodologia de um estudo de caso, sem pretensões de generalização para populações que não sejam as que cursaram o estudo integrado de Programação e participaram da pesquisa. Por isso, não vemos ameaças à validade externa neste trabalho.

\section{Conclusões}

Este artigo teve como objetivo avaliar a motivação de estudantes de um curso de Engenharia de Computação em um estudo integrado de programação orientada a objetos (CS2), que utilizou uma metodologia de aprendizagem baseada em problemas (PBL), replicando um estudo feito anteriormente no mesmo curso de graduação.

Os resultados demonstraram que, no módulo integrador, a Relevância obteve melhores resultados, provavelmente explicado pelos participantes da pesquisa serem majors e considerarem a matéria importante para a sua formação. A Confiança obteve os menores resultados, fato que pode ser explicado pela mudança de paradigma de 
programação de imperativo procedural para orientado a objetos, o que, para muitos, pode ser considerado um processo complicado. Além disso, todos os escores ARCS atingiram mediana acima da neutralidade, o que sugere que os problemas resolvidos pelos estudantes foram razoavelmente bem elaborados e bem recebidos. Em relação aos módulos teóricos, o módulo de Estruturas de Dados obteve os melhores resultados em Relevância, Confiança e Satisfação, sugerindo que a motivação dos estudante pode ter aumentado por a matéria ser um pouco mais prática que as demais.

Pode-se perceber que, apesar da migração do paradigma procedural para o orientado a objetos e das dificuldades que ela pode acarretar, os problemas do MI de Programação e as metodologias utilizadas pelos professores nos módulos teóricos puderam influenciar no processo de aprendizagem dos estudantes, motivando-os ou desmotivando-os em maior ou menor grau. Os resultados sugerem que os estudantes se sentiram motivados em todos os problemas do MI, o que pode ser inferido por conta dos escores ARCS de motivação obtidos.

Trabalhos futuros devem lidar com uma análise mais qualitativa dos aspectos de motivação que ocorrem no contexto de cada um dos módulos teóricos e em cada um dos problemas trabalhados. Além disso, pretende-se comparar este caso com o caso anterior que este estudo procura replicar.

\section{Referências}

Angelo, M. F., Loula, A. C., Bertoni, F. C. \& Santos, J. A. M. (2014). Aplicação e Avaliação do Método PBL em um Componente Curricular Integrado de Programação de Computadores. Revista de Ensino de Engenharia, 33(2).

Arshad, N. (2009). Teaching Programming and Problem Solving to CS2 Students Using Think-Alouds. In Proceedings of the 40th ACM Technical Symposium on Computer Science Education, pp. 372-376.

Bergin, S. \& Reilly, R. (2005). The Influence of Motivation and Comfort-Level on Learning to Program.

Cintra, C.S. \& Bittencourt, R. A. (2015). Being a PBL Teacher in Computer Engineering: An Interpretative Phenomenological Analysis. In 2015 IEEE Frontiers in Education Conference (FIE). IEEE.

Jenkins, T. (2002). On the Difficulty of Learning to Program. In Proceedings of the 3rd Annual Conference of the LTSN Centre for Information and Computer Sciences, pp. 53-58.

Keller, J. M. (2009). Motivational Design for Learning and Performance: The ARCS Model Approach. Springer Science \& Business Media.

Kölling, M. (1999). The Problem of Teaching Object-Oriented Programming, Part 1: Languages. Journal of Object-oriented programming, 11(8): 8-15.

Ribeiro, A. L., Bittencourt, R. A. \& Santana, B. L. (2018). Análise da Motivação em um Estudo Integrado de Programação Baseado em PBL. In Anais do XXVI Workshop sobre Educação em Computação. SBC. 